\title{
Ionic State Relaxation Processes in VUV-Excited Polyatomic Molecules
}

\author{
SYDNEY LEACH† and GÉRALD DUJARDIN \\ Laboratoire de Photophysique Moléculaire du C.N.R.S., $¥$ Bâtiment 213, \\ Université Paris-Sud, 91405 Orsay Cedex, France
}

Advantages of molecular ions for the study of electronic radiationless transitions are discussed. Several coincidence techniques for measuring key relaxation parameters are briefly described. The different cases of radiationless transition studies of molecular ions are outlined and examples mentioned. Coincidence measurements between threshold photoelectrons and ion fluorescence photons emitted by VUV-excited hexafluorobenzene are presented. The radiative, $k_{r}$, and nonradiative, $k_{n}$, relaxation rates for specific vibrational levels in the $\tilde{B}^{2} A_{2 u}$ and $\tilde{C}^{2} B_{2 u}$ states of $C_{6} F_{6}^{+}$are derived from the measured lifetimes and quantum yields of ion fluorescence. The $k_{r}$ rate for excitation of the $\tilde{C}, 0^{\circ}$ level is found to be about $25 \%$ of that for the $\tilde{B}^{2} A_{2 u}$ state where $k_{r}=(18 \pm 3) \times 10^{6} \mathrm{~s}^{-1}$. The $k_{n r}$ rates for the $1^{n}$ and $1^{n} 2^{1}$ progressions in the $\tilde{B}$ state increase with $n$ but exhibit a mode selective behavior. Model calculations of $k_{n r}$ confirm the mode selectivity. These calculations were carried out on a noncommunicating, harmonic oscillator basis. Progressive deviations between experimental and calculated results are interpreted as indicating the gradual onset of vibrational redistribution with increasing vibrational energy. The results illustrate a new method for demonstrating the growing in of intramolecular vibrational relaxation.

\section{INTRODUCTION}

Excited electronic states of molecular ions can relax by three different processes: (1) radiative transitions to lower electronic states; (2) isoenergetic radiationless transitions to bound states; (3) isoenergetic radiationless transitions to dissociation continua. Collisions can

$\dagger$ Also at Départment d'Astrophysique Fondamentale, Observatoire de ParisMeudon, 92190 Meudon, France.

‡ Laboratoire associé à l'Université Paris-Sud. 
modify these processes and give rise to new relaxation pathways, including reactive channels.

Radiationless processes involving the coupling between two or more electronic states we will term electronic nonradiative transitions (ENRT). Intra- and intermolecular relaxation processes can also involve vibrational redistribution. Our discussion will be limited to the collisionless cases of ENRT and vibrational nonradiative transitions (VNRT).

The theory of radiationless transitions has been extensively developed over the past few years; ${ }^{1}$ the models and applications relate mainly to neutral species. Relatively little has been done to study these processes in molecular ions, although such transitions have often been invoked to account for ionic fragmentation. For example, in the quasi-equilibrium theory of mass spectra ${ }^{2}$ it is assumed that molecular ions in electronic excited states undergo ENRT and rapidly convert their electronic energy into vibrational energy of the ground state. This can subsequently lead to vibrational predissociation if sufficient vibrational energy is available, and the intramolecular dynamics (VNRT) allows the ion to attain a dissociative configuration faster than any competitive process that tends to deactivate the high vibrational levels of the ground state. However, as discussed below, there are specific advantages of molecular ions as objects for the study of radiationless transitions. ${ }^{3}$ In Section 2 we outline these advantages. Section 3 presents the techniques for measuring relaxation parameters, in particular ion fluorescence decay lifetimes and quantum yields, using coincidence techniques. The different cases of radiationless transitions in molecular ions are briefly presented in Section 4 and some examples mentioned. Section 5 is devoted to a particular example: ENRT and VNRT in excited electronic states of the hexafluorobenzene cation studied by the technique of coincidence measurements between threshold photoelectrons and ion fluorescence photons, and by model calculations.

\section{ADVANTAGES OF MOLECULAR IONS FOR THE STUDY OF RADIATIONLESS TRANSITIONS}

In radiationless transition studies, the quantities determined experimentally are quantum yields and time-resolved decay patterns of light 
emission and/or fragmentation. With respect to neutral species, the study of molecular ions presents a number of advantages. These derive from two essential sets of properties, one structural, the other instrumental.

\subsection{Structural advantages}

Many molecular ions are odd electron, open shell species, so that the electronic states of interest are doublet $(D)$ and quartet $(Q)$ states. The energy separation $\Delta E\left(D_{1}-D_{0}\right)$ between the electronic ground state $D_{0}$ and the first excited state of the same multiplicity, $D_{1}$, is of ten rather less than the parent molecule value $\Delta E\left(S_{1}-S_{0}\right)$ involving singlet states. ${ }^{3}$ The lowest dissociation pathway in parent and ion are similar in energy in many cases. It follows that the $D_{1}$ state will tend to lie below the flrst dissociation limit of the ion, whereas in closed shell species $S_{1}$ often lies in the vicinity of the lowest dissociation threshold, so that predissociation of all or some of the vibrational levels of $S_{1}$ can occur. A further point resulting from the relation $\Delta E\left(D_{1}-D_{0}\right)<\Delta E\left(S_{1}-S_{0}\right)$ is that the $D_{1}-D_{0}$ electronic transition will often occur in a spectral region that is more accessible than $S_{1}-S_{0}$ from the viewpoint of excitation (selective or otherwise) and detection of photon emission.

Another structural advantage of molecular ions is particularly relevant to the application of radiationless transition theories, and provides a simplification with respect to closed shell species. This derives from the fact that the excited $S_{1}$ state can be coupled to vibronic levels of at least two lower-lying electronic states, viz. the first excited triplet state (and often also to higher triplets) $T_{1}$ and the electronic ground state $S_{0}$, whereas in many open shell molecular ions the vertical excitation energy $\Delta E_{v e r t}\left(Q_{1}-D_{0}\right)$ of the lowest lying quartet state $Q_{1}$ will be greater than that of the first excited doublet state $\Delta E_{v e r t}\left(D_{1}-\right.$ $\left.D_{0}\right)$. Radiationless transitions from $D_{1}$ can therefore only occur by coupling to only one lower lying state, the electronic ground state $D_{0}$ (Figure 1).

These various structural advantages have made it possible to study the radiationless decay rates of excited electronic states of molecular ions over a much greater range of internal vibrational energy ${ }^{4-6}$ than is often possible for neutral species. ${ }^{1}$ 

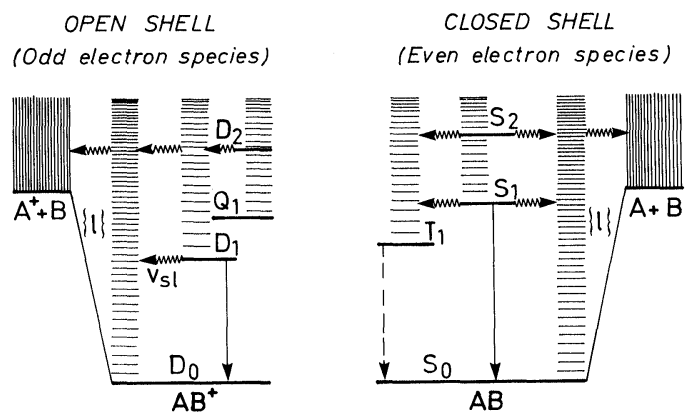

FIGURE 1 Schematic comparison between electronic level manifolds, interelectronic state coupling and dissociation limits of open shell and closed shell molecular species. $v_{s l}$ is the matrix element coupling the $|s\rangle$ vibronic level of the excited doublet state $D_{1}$ to isoenergetic vibrational levels $\{|l\rangle\}$ of the $D_{0}$ ground state; similar matrix elements are posited for coupling between other open shell electronic states or between closed shell states.

\subsection{Instrumental advantages}

The existence of an electric charge on the ion makes the latter relatively easy to detect, count and identify. Photoionization will create not only an ion but also a photoelectron (except in the case of ion pair formation) of well defined kinetic energy, which can be used as an energy probe. Charged particle detection can proceed by means of ion multipliers, electron multipliers, channel plates, etc., so that the number of ionic species can be counted; determination of their nature requires some form of mass spectrometry, by magnetic and/or electric field ion dispersion or by mass dependent time-offlight (TOF) techniques. Ion-photon and electron-photon coincidence methods can be used to determine ion fluorescence lifetimes and quantum yields; fragmentation yields can be measured by ion-electron coincidence counts (Table I). The study of selected vibronic states is possible with these methods. The techniques used for equivalent measurements in neutral species are much less direct. ${ }^{15}$

\section{MEASUREMENT OF RELAXATION PARAMETERS OF MOLECULAR IONS USING COINCIDENCE TECHNIQUES}

Ion level energies are displayed in bands of the photoelectron spectrum (PES) in which fixed wavelength photon excitation is used and 


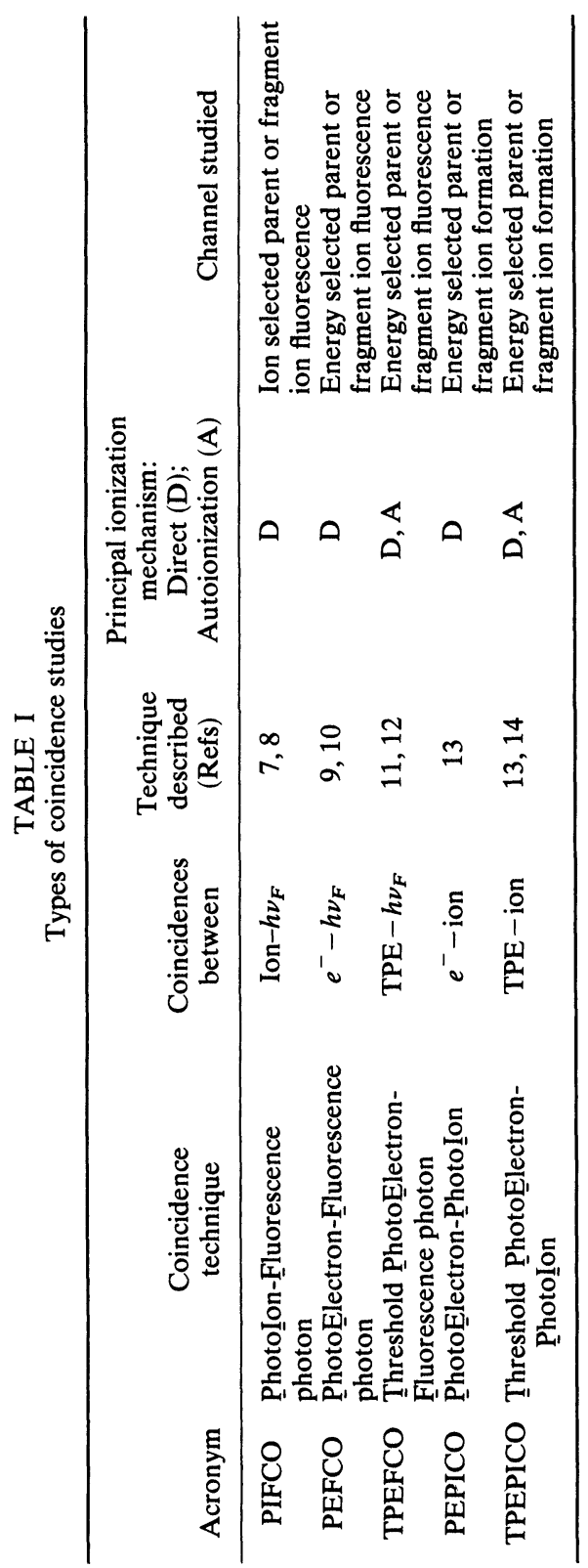


measurement is made of the variable kinetic energy of the photoelectrons (Figure 2). The energy resolution depends on the linewidth of the exciting radiation, the translational motion of the absorbing species and the resolving power of the electron energy analyzer. Usual energy resolution in PES is $20 \mathrm{meV}$ but this can be improved with care. Figure 2 also illustrates threshold photoelectron spectroscopy (TPES) in which a variable photon energy source is used but only zero-kinetic energy photoelectrons (threshold photoelectrons) are detected. The states detected therefore have the same energy as that of the absorbed photon. Threshold photoelectron spectroscopy can thus provide information on both direct and auto-ionization processes. ${ }^{16}$ Comparison with conventional PES enables one to separate the threshold photoelectron signal into its direct ionization and autoionization components. ${ }^{12}$

Three types of coincidence studies have been made to measure ion fluorescence decay behaviour and quantum yields:

1) Photoion-fluorescence photon coincidences (PIFCO);

2) Photoelectron-fluorescence photon coincidences (PEFCO);

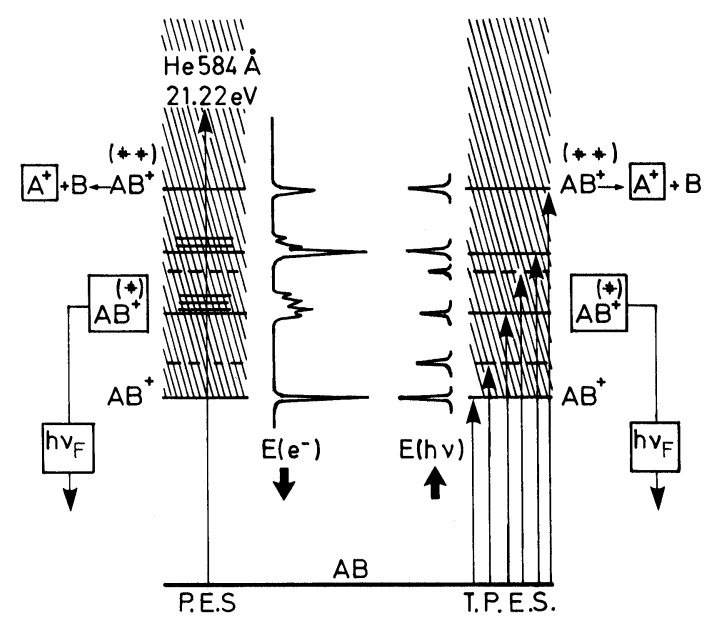

FIGURE 2 PES = Photoelectron spectroscopy using fixed photon excitation energy and measuring the variable kinetic energy of the photoelectrons. TPES $=$ Threshold photoelectron spectroscopy using variable photon excitation energy and measuring fixed (zero) kinetic energy photoelectrons. Dashed lines represent superexcited states of $A B$. 
3) Threshold photoelectron-fluorescence photon coincidences (T-PEFCO).

With the PIFCO technique, measurement is made of the average fluorescence lifetime $\bar{\tau}$ and quantum yield $\bar{\phi}_{F}$; the average is over the occupied vibrational levels of a particular emitting electronic state of the ion. The PEFCO and T-PEFCO techniques allow $\tau$ and $\phi_{F}$ measurements to be made at specific excitation energy levels within the resolution of the particular method $\left(\approx 100 \mathrm{meV}\right.$ for $\mathrm{PEFCO}^{10}$ and $\approx 40 \mathrm{meV}$ for T-PEFCO. ${ }^{12}$

The radiative $\left(k_{r}\right)$ and nonradiative $\left(k_{n r}\right)$ rates can be calculated using the following relations:

$$
k_{r}=\phi_{F} \tau^{-1} \quad k_{n r}=\left(1-\phi_{F}\right) \tau^{-1}
$$

Ion fragmentation can be studied using other coincidence techniques, in addition to the PIFCO method:

4) Photoelectron-photoinon coincidences (PEPICO);

5) Threshold photoelectron-photoion coincidences (T-PEPICO).

These five coincidence techniques are described in the references cited in Table I. A compilation of references to PIFCO, PEFCO and T-PEFCO work and results (up to June, 1982) can be found in Table V of Ref. 16.

We stress that the threshold photoelectron (TPE) techniques allow studies to be made of coincidences between TPE and the products of superexcited state autoionization channels such as parent ions, fragment ions, fluorescence of excited ions, etc. ${ }^{12}$ Further useful information on the autoionization processes can be obtained from measurement of the photoelectron energy distribution at specific excitation energies.

\section{RADIATIONLESS TRANSITION STUDIES ON MOLECULAR IONS: GENERAL REMARKS}

The development and status of radiationless transition theory have been extensively reviewed in the last few years. ${ }^{1}$ Aspects of the theory useful in the study of molecular ions have been discussed elsewhere ${ }^{3,16}$ 
in terms of the three cases commonly distinguished as the sparse level "resonance limit," intermediate case, and statistical limit.

In the sparse level "resonance limit," the optically excited zero-order state $|s\rangle$ is coupled nonradiatively to one or a handful of combining states $\{|l\rangle\}$. Since we can generally neglect spin-orbit effects in considering the relaxation of the first excited state of odd-electron molecular ions, the matrix element $v_{s l}$ involves the nuclear kinetic energy operator. In the sparse level case, each linear combination of interacting levels will correspond to different $s-l$ energy intervals and have a distinct $v_{s l}$ value, so that each molecular eigenstate will emit with its own characteristic decay time. An example of a molecular ion which has been studied in terms of sparse level radiationless transitions is $\mathrm{CO}_{2}^{+}$. This has involved both high resolution spectroscopy, ${ }^{17,18}$ and fluorescence quantum yield and lifetime measurements, ${ }^{19}$ including $\phi_{F}$ and $\tau$ measurements on individual rotational levels. ${ }^{20}$

In the intermediate case, the density of $\{|l\rangle\}$ states is of the order $10^{3}-10^{6}$ states per $\mathrm{cm}^{-1}$. In the coarse-grained approach to which present theory has accustomed us, the $\{|l\rangle\}$ states can be considered to consist of two classes, $\left\{\left|l_{c}\right\rangle\right\}$ and $\left\{\left|l_{d}\right\rangle\right\}$ which are coupled to $|s\rangle$ with matrix elements that bear the relation $v_{s c} \gg v_{s d}$. Interpretation of experimental results is usually made on the basis of the strong coupling limit, which implies that $v_{s c}$ significantly exceeds $\rho^{-1}$, the average spacing between successive $l_{c}$ levels, and on the following assumptions: constant $v_{s c}$, equal spacing between successive energy levels $l_{c}$, and constant level width $\gamma_{c}$. An experimental characteristic of the intermediate case is the observation of multiexponential fluorescence decay. This has been observed and studied for the monochloroacetylene ion. ${ }^{21}$ Going beyond the coarse-grained model will become necessary when experimental techniques in studies of radiationless transitions in polyatomic ions have sufficiently progressed in energy resolution.

In the statistical limit, the density of $\{|l\rangle\}$ states isoenergetic with $|s\rangle$ is very high (Figure 3), so that they form a uniform quasicontinuum. Typical values are $\rho_{l}=10^{10} \simeq 10^{20}$ states $/ \mathrm{cm}^{-1}$. This is the case in polyfluorobenzene cations for which the PIFCO results of Dujardin et al $^{8}$ have previously shown that ENRT corresponds to the so-called large molecule case of radiationless transitions. In this, ENRT from the vibronic $|s\rangle$ state of energy $E_{s}$ corresponds to 


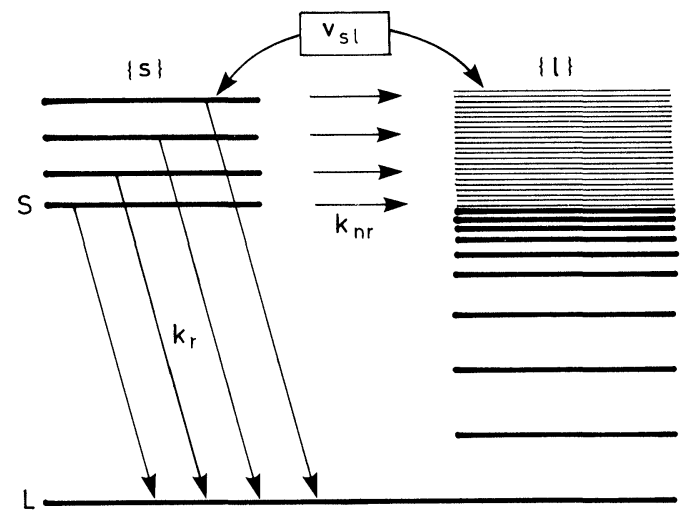

FIGURE 3 Vibronic coupling scheme in the statistical limit of radiationless transitions. $k_{r}$ and $k_{n r}$ are respectively the radiative and nonradiative relaxation rates for coupling between the excited electronic state $S$ and the ground state $L .\{s\}$ and $\{l\}$ are vibrational levels of $S$ and $L$ respectively. $v_{s l}$ is the nonradiative coupling matrix element.

an irreversible transition to quasi-isoenergetic $\{|l\rangle\}$ states (energy $E_{l}$ ) with a rate given by:

$$
k_{s-l}\left(=k_{n r}^{s}\right)=\frac{2 \pi}{\hbar} \sum_{l} v_{s l}^{2} \delta\left(E_{s}-E_{l}\right)
$$

To the radiative width $\gamma_{r}^{s}$ of the $|s\rangle$ state is added a nonradiative term $\gamma_{n r}^{s}$ which derives from $s-l$ coupling. The fluorescence quantum yield is then given by:

$$
\phi_{F}=\gamma_{r}^{s} /\left(\gamma_{r}^{s}+\gamma_{n r}^{s}\right)
$$

and will therefore tend to be less than unity in the statistical limit. Furthermore, in this case, the nonradiative rate $k_{n r}^{s}=\gamma_{n r}^{s} / \hbar$ is related to $v_{s l}$ and $\rho_{l}$ by the Fermi-Wentzel Golden Rule expression which is derived from Eq. (2) when $v_{s l}$ varies slowly enough to be represented by its average value. (Henceforward, $v_{s l}$ is considered to be this average value):

$$
k_{n r}^{s}=2 \pi \hbar \rho_{l} v_{s l}^{2}
$$

and the decay of the $|s\rangle$ state will then be monoexponential:

$$
\tau_{s}=\hbar\left(\gamma_{r}^{s}+\gamma_{n r}^{s}\right)^{-1}=\left(k_{r}^{s}+k_{n r}^{s}\right)^{-1}
$$


We denote $E_{s}=E_{0}+E_{v}$ as the total energy of the $|s\rangle$ state where $E_{0}$ is the electronic energy of the initial optically excited electronic state relative to the final electronic state and $E_{v}$ is the vibrational energy in the initial electronic state.

It has been shown ${ }^{1}$ that $k_{n r}$ can be expressed as an exponentially decreasing function of the electronic energy gap $E_{0}$ (energy gap law), which we will call the first general law of ENRT. This law is derived from Eq. (4) on the basis of a number of approximations (in particular, that $v_{s l}$ can be written as a single product of an electronic factor and a vibrational overlap integral), and it should be valid when the energy gap $E_{0}$ is large compared with the vibrational quantum of an accepting mode.

The electronic energy gap cannot, of course, be varied experimentally for a single species. However, it is possible to compare the members of a class of related species with different $E_{0}$ values but for which the electronic part of $v_{s l}$ is expected to be approximately constant. This was done by Leach et al. ${ }^{3}$ using the mean values $\bar{\phi}_{F}(\tilde{B})$ and $\bar{\tau}(B)$ measured for the occupied $\tilde{B}$ state vibronic levels of eight polyfluorobenzene cations. Although, as discussed by Dujardin et al., ${ }^{8}$ many intrinsic factors can change from one fluorobenzene cation to another, an approximate exponential decrease of $k_{n r}$ with increasing $E_{0}$ was observed experimentally. ${ }^{3}$ We remark that this behaviour is related to the decrease of the Franck-Condon factors derived from $v_{s l}$ in Eq. (4); the greater is $E_{0}$, the larger the necessary vibrational energy changes from $|s\rangle$ to $|l\rangle$ and the smaller is the overlap of vibrational functions.

The rest of the present work is concerned more particularly with what we call the second general law of ENRT in "large molecules." This predicts an increase of $k_{n r}\left(E_{0}+E_{v}\right)$ when the vibrational energy $E_{v}$ increases, and is expected to be valid for moderate and large values of the electronic energy gap. ${ }^{1}$

We remark that the present study does not concern cases where dissociative relaxation pathways are competitive with ion fluorescence. Reference to such cases is made elsewhere. ${ }^{16}$ Our concern is with the "large molecule" ion $\mathrm{C}_{6} \mathrm{~F}_{6}^{+}$for which a PEFCO study was previously carried out by Maier and Thommen. ${ }^{10}$ The better time and energy resolutions in our T-PEFCO experiments, coupled with the possibility of populating ion levels in the Franck-Condon gaps, via autoionization, made it possible to make direct determinations of 
$\phi_{F}$ and $\tau$ for a greater number of levels than in the PEFCO work. A number of important new features were revealed, as will be discussed later.

\section{ELECTRONIC AND VIBRATIONAL RADIATIONLESS TRANSITIONS IN EXCITED ELECTRONIC STATES OF $\mathrm{C}_{6} \mathrm{~F}_{6}^{+}$}

\subsection{Experimental: TPES and T-PEFCO techniques}

The experimental technique for obtaining threshold photoelectron spectra (TPES) and for counting coincidences between threshold photoelectrons and the fluorescence photons (T-PEFCO) emitted by the $\mathrm{C}_{6} \mathrm{~F}_{6}^{+}$excited into selected vibronic levels has been described in detail. ${ }^{12}$ We recall here only the salient features of the experiment.

The photon source is synchrotron radiation from the Orsay storage ring (ACO), dispersed by a $1 \mathrm{~m}$ normal incidence grating ( 2400 lines $/ \mathrm{mm}$ ) McPherson monochromator. This continuously tunable light source was operated with a band pass of $2 \AA$. Photoelectrons produced by photoionization of a jet of $\mathrm{C}_{6} \mathrm{~F}_{6}\left(p \sim 5 \times 10^{-4}\right.$ torr $)$ are accelerated towards a time-of-flight electron spectrometer by a $1.4 \mathrm{~V} / \mathrm{cm}$ electric field and are detected by a microchannel plate electron multiplier. The kinetic energy spectrum of the photoelectrons is determined by measurement of the time interval between a synchrotron radiation pulse $(1.2 \mathrm{~ns}$ FWHM, $13.6 \mathrm{MHz})$ and detection of the photoelectrons. Threshold photoelectrons (TPE) can therefore be detected by gating the electron detector after each synchrotron radiation pulse with a time window centered on the specific arrival time of the zero kinetic energy photoelectrons. We recall that our electron energy resolution is better than $45 \mathrm{meV}$. The time-of-flight analysis requires a number of calibrations and corrections which are detailed elsewhere. ${ }^{12}$ Threshold photoelectron spectra are obtained by counting the TPE as a function of the photon excitation energy.

At certain excitation energies, the $\mathrm{C}_{6} \mathrm{~F}_{6}^{+}$emits fluorescence photons mainly corresponding to the $\tilde{B}^{2} A_{2 u}-\tilde{X}^{2} E_{1 g}$ transition in the $400-$ $600 \mathrm{~nm}$ spectral region. ${ }^{23} \mathrm{~T}$-PEFCO experiments are done as follows. Coincidence detection and counting are carried out using a time-toamplitude converter whose start input and stop input are respectively the TPE signal and the fluorescence photon signal. The latter was detected by an R943 Hamamatsu photomultiplier with appropriate 
electronics. The coincidence signals are stored in a multichannel analyzer. The time scale is calibrated by the method used by Dujardin et $a l^{8}{ }^{8}$ The lifetime $\tau$ of the energy selected state is determined by fitting the accumulated coincidence curve to an exponential decay curve using a least-squares method. The total number of true coincidences $N_{c}$ during the time of the coincidence count is obtained by time integration of the T-PEFCO curve after subtraction of the false coincidence background. During the same time interval we count $N_{e}$, the total number of threshold photoelectrons. The TPE can result from direct ionization producing the emitting level of $\mathrm{C}_{6} \mathrm{~F}_{6}^{+}$, e.g., $\tilde{B}^{2} A_{2 u}(v)$, and also from autoionization processes which can form not only the fluorescing state of the ion but also nonradiative isoenergetic vibrational levels belonging to lower electronic states, notably the $\tilde{X}^{2} E_{1 \mathrm{~g}}$ ground state. $^{12}$

Methods for obtaining the branching ratios for these processes in $\mathrm{C}_{6} \mathrm{~F}_{6}^{+}$are described in detail elsewhere. ${ }^{12}$ These methods enable us to determine $N_{e}\left(j, v^{\prime}\right)$ the number of TPE corresponding to formation of the $j, v^{\prime}$ state of the ion. We remark that with the T-PEFCO technique, autoionization processes can increase the relative number of ions formed at certain $j, v^{\prime}$ levels with respect to the PEFCO technique in which only direct ionization processes are measured. This is particularly true in the so-called Franck-Condon gaps; the T-PEFCO technique therefore permits us to measure the fluorescence decay characteristics of $j, v^{\prime}$ levels whose access is difficult with the PEFCO technique.

The fluorescence quantum yield $\phi_{F}\left(j, v^{\prime}\right)$ is then determined from the relation:

$$
\phi_{F}\left(j, v^{\prime}\right)=N_{c} / N_{e}\left(j, v^{\prime}\right) \cdot f_{h \nu}
$$

where $f_{h \nu}$ is the fluorescence photon detection efficiency whose method of determination is described elsewhere. ${ }^{3,8}$

\subsection{Experimental results}

$\mathrm{C}_{6} \mathrm{~F}_{6}^{+}$is a hexagonal species of $D_{6 h}$ nuclear symmetry. The three electronic states of concern to us are the ground $\tilde{X}^{2} E_{1 \mathrm{~g}}(9.930 \mathrm{eV})$, and excited $\tilde{B}^{2} A_{2 u}(12.595 \mathrm{eV})$ and $\tilde{C}^{2} B_{2 u}(13.880 \mathrm{eV})$ states (Figure 4). Their adiabatic energies given in parentheses were determined from TPES. ${ }^{12}$ Vibrational levels in the nondegenerate electronic 


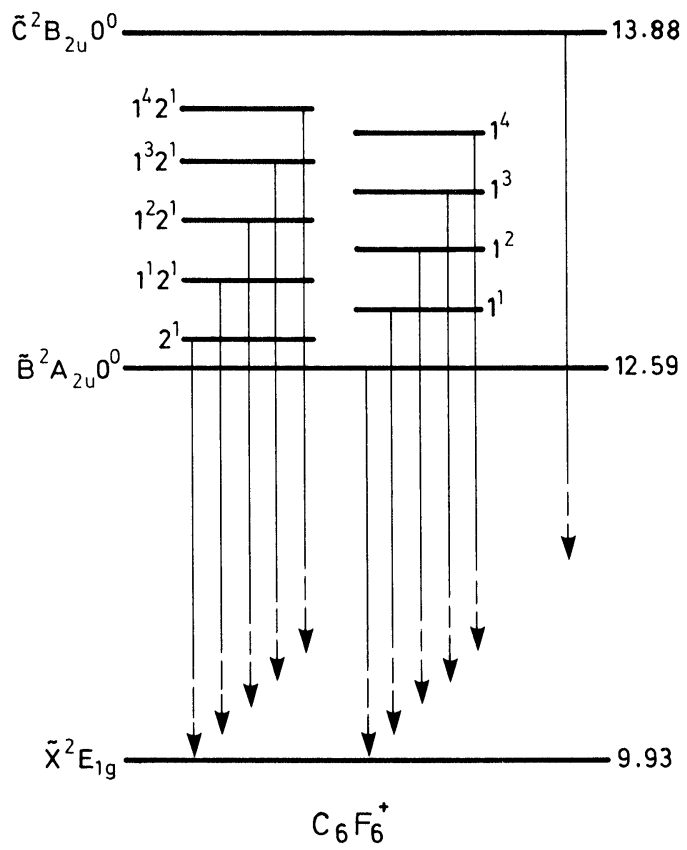

FIGURE 4 Electronic states and some of the vibrational levels involved in a T-PEFCO study of excited state relaxation in $\mathrm{C}_{6} \mathrm{~F}_{6}^{+}$. The lowest dissociation threshold is at $15.3 \mathrm{eV} .{ }^{12}$

excited states populated by photoionization involve totally symmetric modes of which there are two in this ion: $\nu_{1}\left(a_{1 g}\right)$ which is a C-F stretching mode, and $\nu_{2}\left(a_{1 \mathrm{~g}}\right)$, a ring vibration. We note that, as monitored in the TPES, ${ }^{12}$ the $\mathrm{C}_{6} \mathrm{~F}_{6}$ photoionization process involves considerable autoionization from superexcited states to high vibrational levels of the ground state and to $\tilde{B}$ state vibrational levels. This occurs in Franck-Condon gaps as well as in Franck-Condon regions. Autoionization thus provides a mechanism for formation of certain $\mathrm{C}_{6} \mathrm{~F}_{6}^{+}$levels which are not accessible by direct ionization. ${ }^{12,24}$

Measurement of $\phi_{F}\left(v^{\prime}\right)$ and $\tau\left(v^{\prime}\right)$ was made for selected vibrational levels (Figure 4) using the T-PEFCO technique as described in detail elsewhere. ${ }^{12}$ The radiative and nonradiative rates, respectively $k_{r}\left(v^{\prime}\right)$ and $k_{n r}\left(v^{\prime}\right)$, were derived from the relations given in Eq. (1). The $k_{r}$ 
results are presented in Figure 5 and the $k_{n r}$ in Figure 6. In each case the data are compared with the results of Maier and Thommen. ${ }^{10}$

$k_{r}$ rates There is excellent agreement between our T-PEFCO results and the PEFCO data of Maier and Thommen for common excitation energies (Figure 5). We note that the time resolution $(\approx 15 \mathrm{~ns})$ in the PEFCO measurements was insufficient to determine the lifetime of the $\tilde{C}\left(0^{\circ}\right)$ level. In the T-PEFCO experiments our time resolution was $\sim 1$ ns so that we were able to directly measure the $\tilde{\mathrm{C}}\left(0^{\circ}\right)$ level and show that its $k_{r}$ rate is about $25 \%$ of that of the $\tilde{B}\left(0^{\circ}\right)$ state $\left[k_{r}(\tilde{B})=(18 \pm 3) \times 10^{6} \mathrm{~s}^{-1}\right]$.

We remark that Maier and Thommen ${ }^{10}$ determined the nonradiative $k_{n r}(\tilde{C})$ rates from the relation $k_{n r}=k_{r}\left(1-\phi_{F}\right) / \phi_{F}$ where $k_{r}(\tilde{C})$ was taken as being equal to $k_{r}(\tilde{B})$. Our results show that this assumption is incorrect and so invalidates the $k_{n r}$ results of Maier and Thommen in the $\tilde{C}$ state region. The nature of the fluorescence emission in coincidence with $\tilde{C}$ state excitation has been discussed in detail. ${ }^{6}$

$k_{n r}$ rates The experimental values of the nonradiative rate $k_{n r}$ in the $\tilde{B}$ state region are plotted in Figure 6 as a function of internal vibrational energy $E_{v}$. This figure also includes the $k_{n r}$ data reported by Maier and Thommen ${ }^{10}$ for this region. The results of the latter

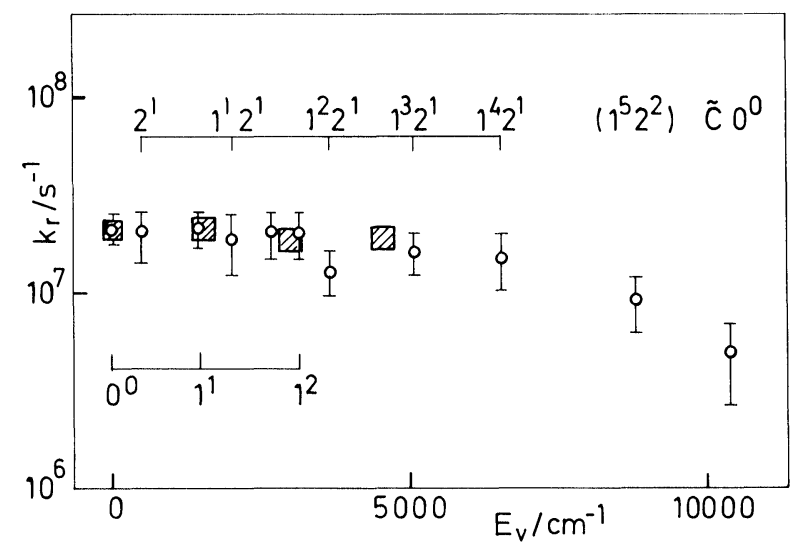

FIGURE 5 Radiative decay rate $k_{r}$ of $\tilde{B}, v^{\prime}$ and $\tilde{C}, 0^{\circ}$ levels. $\bigcirc$ represent T-PEFCO values. $^{6}$ represent PEFCO values ${ }^{10}$ whose error limits $\left( \pm 2 \times 10^{6} \mathrm{~s}^{-1}\right)$ are not shown. 


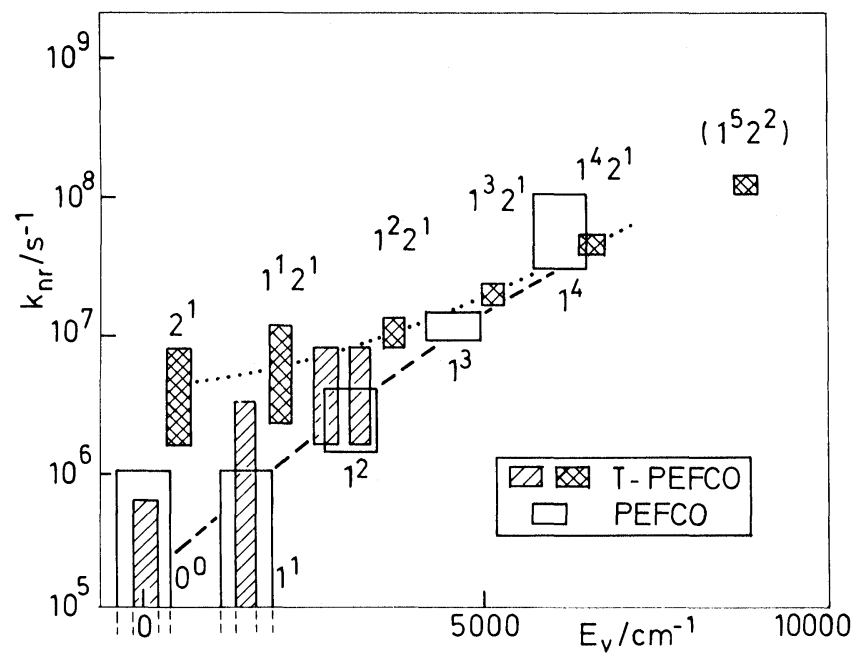

FIGURE 6 Nonradiative decay rate $k_{n r}$ of the $\tilde{B}, v^{\prime}$ levels of $\mathrm{C}_{6} \mathrm{~F}_{6}^{+}$. Rectangles indicate the uncertainty limits of T-PEFCO ${ }^{6}$ and PEFCO ${ }^{10}$ measurements. Data on levels involving mode 2 excitation are given by cross-hatched rectangles.

concern essentially the $0^{\circ}$ level and the $1^{n}$ progression, (although in the PEFCO excitation technique some small overlap with $1^{n} 2^{1}$ levels may exist due to the $100 \mathrm{meV}$ energy resolution) whereas our $\mathrm{T}$ PEFCO measurements were made for the $1^{n} 2^{1}$ progression as well as $0^{\circ}$ and $1^{n}$, with a resolution better than $45 \mathrm{meV}$. The agreement is good between the PEFCO and T-PEFCO results for the common values of $E_{v}$.

Our two principal observations are as follows:

1) We note first of all that the $1^{n}(n=0-4)$ and $1^{n} 2^{1}(n=0-4)$ progressions, taken separately, each exhibit a monotonic, quasiexponential, increase in $k_{n r}$ as $E_{v}$ increases.

2) Figure 6 shows that the $k_{n r}$ rate depends not only on the vibrational energy of the optically excited $j, v^{\prime}$ state but also on the specific optical vibrational mode excited. This type of behaviour has previously been observed in neutral species e.g., benzene. ${ }^{1,25}$ In the case of $\mathrm{C}_{6} \mathrm{~F}_{6}^{+}$it is clear from Figure 6 that the $k_{n r}$ rate is enhanced, with respect to the $1^{n}$ progression, when the $\nu_{2}$ mode is excited simultaneously with $1^{n}$ in the $1^{n} 2^{1}$ combinations. 
Most of the rest of this paper is devoted to interpretation of these two results. This involves some model calculations of the relative nonradiative rates of vibrational levels of the $\tilde{B}$ state.

\subsection{Relative nonradiative rates of the $\tilde{B}$ state vibrational levels of $\mathrm{C}_{6} \mathrm{~F}_{6}^{+}$: results of model calculations and comparison with experiment}

The starting point of model calculations of the relative $k_{n r}\left(v^{\prime}\right)$ values is Eq. (2). The key is in the assumption that the overall interaction energies $\mathrm{v}_{\mathrm{sl}}$ can be factorized into single products of electronic and vibrational factors. The electronic factor, whose calculation would be complicated, is considered to be constant for different vibrational levels of the $\tilde{B}$ state (Condon approximation following from the Born-Oppenheimer (B.O.) approximation). The electronic factor can therefore be factorized out in the relative rate calculations. The latter then reduces to a calculation of Franck-Condon factors taking into account the energy conservation condition represented by the $\delta$-function in Eq. 2.

Calculation of the relative $k_{n r}\left(v^{\prime}\right)$ rates is equivalent to determining the vibrational dependence of $k_{s \rightarrow l}$ from Eq. (2). For this we use the nonintegral occupation number (NION) method which was developed by Kühn $e t$ al. ${ }^{26}$

It enables us to calculate the appropriate Franck-Condon factors with a technique that elegantly solves the delicate problem of ensuring that energy is conserved in the radiationless transition i.e., it deals adequately with the $\delta$-function in Eq. 2 . The NION method has been shown to be consistent with other methods used for relative $k_{n r}$ rate calculations, such as the "saddle point approximation," but is of simpler application.

Details of the calculation for $\mathrm{C}_{6} \mathrm{~F}_{6}^{+}$are presented elsewhere $;^{6}$ only the results are given here, in graphical form, in Figure 7. Both calculated and experimental non-radiative rates increase monotonically with $E_{v}$ for each vibrational progression $1^{n}$ and $2^{1} 1^{n}(n=0-4)$ taken separately. This is as expected from the "second law of ENRT" when the electronic energy gap is large. ${ }^{22}$ Furthermore, the calculated results predict that $k_{n r}$ is mode-sensitive in agreement with our experimental finding that whenever $\nu_{2}$ is optically excited the nonradiative rate is enhanced. This is most apparent at low values of the excess 


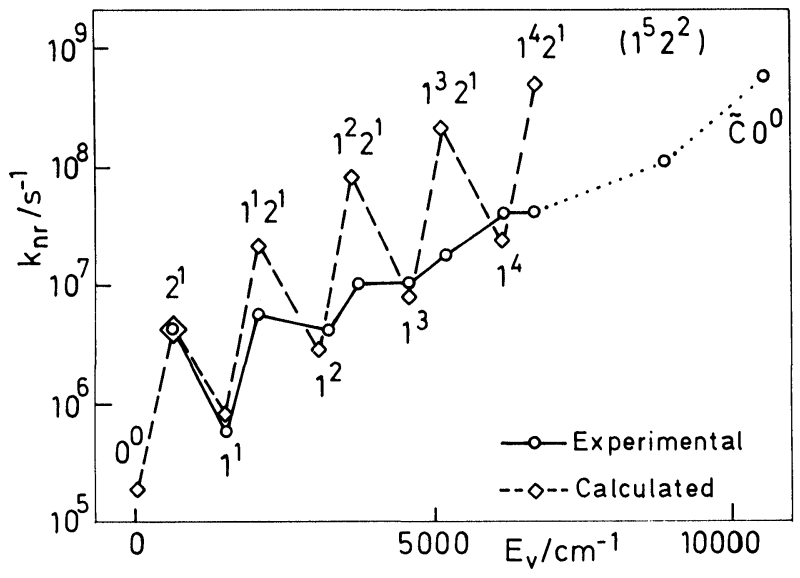

FIGURE 7 Comparison of experimental and calculated nonradiative relaxation rates $k_{n r}$ of selected levels $\tilde{B}, v^{\prime}$ of $\mathrm{C}_{6} \mathrm{~F}_{6}^{+}$. ${ }^{+}$Calculated values are normalized to the experimental value $k_{n r}\left(2^{1}\right)$.

vibrational energy $E_{v}$. The $k_{n r}$ behavior at increasing values of $E_{v}$ will now be discussed.

\subsection{Vibrational redistribution (VNRT)}

Our calculations were carried out on a harmonic oscillator basis, and thus with the implicit assumption of no vibrational redistribution in the optically excited state $|s\rangle$. However, VNRT processes could be of some importance when their rate is comparable or greater than the ENRT or radiative transition rates. The VNRT rate may be enhanced in the case of "large molecules" because of their high vibrational level densities.

In the present work, the observation of mode selective behaviour of the $k_{n r}$ rates for the first few levels of the $\mathrm{C}_{6} \mathrm{~F}_{6}^{+} \tilde{B}$ state is consistent with the belief that VNRT is not effective in this energy region, or at least that it is very incomplete, as would be expected from the vibrational level densities (e.g., $1.7 \times 10^{3}$ states per $\mathrm{cm}^{-1}$ at $E_{v}=$ $2000 \mathrm{~cm}^{-1}$ ). The calculated points in Figure 7 represent the $k_{n r}$ rates that would be obtained if the excited vibronic level retained complete memory of its initial state during the ENRT process, i.e., if no VNRT occurred. Inspection of Figure 7 shows that as the combination levels 
$1^{n} 2^{1}$ contain increasing amounts of mode 1 , the experimental $k_{n r}\left(1^{n} 2^{1}\right)$ rates increasingly deviate from the corresponding theoretical values and gradually approach the calculated values of $k_{n r}\left(1^{n}\right)$. We interpret this behaviour as indicating that vibrational level interactions among the $|s\rangle$ levels [VNRT (s)] becomes more important as $E_{v}$ grows.

The model represented by Figure 3 is therefore inadequate. A more appropriate model scheme is illustrated in Figure 8. Optically excited vibrational states $\{s\}$ in the excited electronic state $S(\equiv \tilde{B})$ can undergo coupling via matrix element $v_{s s^{\prime}}$ to other quasi-isoenergetic vibrational levels in the $\left\{s^{\prime}\right\}$ manifold of the $S$ state. These can be considered to form nuclear molecular eigenstates $\{\bar{s}\}$, in the excited electronic state, which can couple in turn to isoenergetic highly excited vibrational levels $\{l\}$ of the $L(\equiv \tilde{X})$ ground state. However, it is more useful to continue our discussion in the zero-order basis of $\{s\},\left\{s^{\prime}\right\}$ and $\{l\}$; coupling will be discussed in terms of the matrix elements $v_{s l}$ and $v_{s^{\prime} l}$.

In the present scheme we have no need to consider vibrational redistribution in the $\{l\}$ states. The effect of VNRT $(l)$ would be to add to the level width of the $\{l\}$ states. The density of the $\{l\}$ states isoenergetic with a particular $s$ level is very high $\left(\sim 10^{17} \mathrm{~cm}^{-1}\right.$ accord-

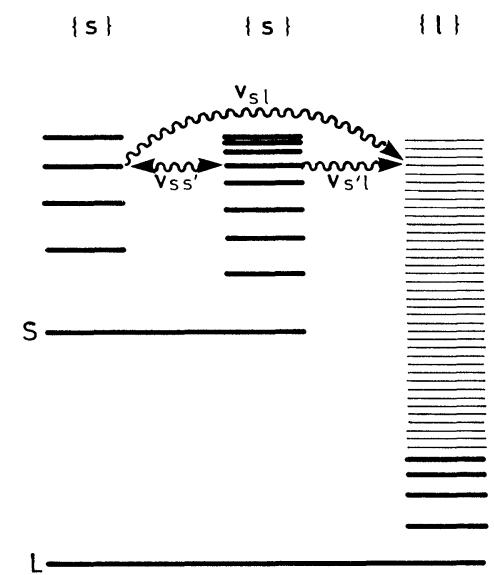

FIGURE 8 Vibronic coupling scheme in the statistical limit including electronic nonradiative transitions [ENRT] via coupling matrix elements $v_{s l}$ and $v_{s^{\prime}}$, and vibrational nonradiative transitions VNRT $(s)$ via coupling matrix elements $v_{s s^{\prime}} .\{s\}$ are the optically excited and $\left\{s^{\prime}\right\}$ other vibrational levels of the $S$ state. 
ing to a Haarhoff-type calculation ${ }^{3}$ ), so that the effective quasicontinuum of $\{l\}$ states would be little affected by the introduction of $\operatorname{VNRT}(l)$.

The operators in the various matrix elements will have the following physical significance:

1) $v_{s s^{\prime}}$ : vibrational anharmonicity and Coriolis (rotationalelectronic) coupling; we may use the term Fermi resonance as a generic name for any interaction which takes place between closelying zero-order vibrational levels and which is caused by terms in the vibrational potential energy expression cubic or higher in the vibrational coordinates.

2) $v_{s l}$ and $v_{s^{\prime} l}$ : nuclear kinetic energy operators resulting from the incomplete Hamiltonian which is implied in deriving the zero-order states. No spin-orbit operator contributions are necessary in our case of open shell molecular ions where the lowest accessible quartet state is above the first, and possibly the second excited doublet state. $v_{s l}$ and $v_{s^{\prime} l}$ correspond to coupling with the quasi-continuum of $\{l\}$ states and thus lead to irreversible transitions whose rates are respectively $k_{s l}$ and $k_{s^{\prime} l \cdot}$

Let us consider successively I, II and III, three different regions of $E_{v}$, corresponding to increasing level density $\rho\left(\tilde{B}, v^{\prime}\right)$. The boundaries between these regions are not rigid. Our experimental results (e.g., Figure 7) lead us to propose as approximate ranges in the $\tilde{B}$ state of $\mathrm{C}_{6} \mathrm{~F}_{6}^{+}: E_{v}(\mathrm{I})=0$ to $\sim 2500 \mathrm{~cm}^{-1}, E_{v}(\mathrm{II})=\sim 2500$ to $\sim 4500 \mathrm{~cm}^{-1}$, $E_{v}$ (III) $>4500 \mathrm{~cm}^{-1}$. From the viewpoint of VNRT(s), these regions correspond respectively to the sparse level (I), intermediate case (II) and statistical limit (III) analogues of ENRT. ${ }^{27-30}$

At low level densities $\rho\left(\tilde{B}, v^{\prime}\right)$ (region I), and in the absence of Fermi resonance and neglecting Coriolis coupling (both of which can lead to mixing of zero-order (deperturbed) levels within the $\tilde{B}$ vibrational manifold), we expect the optically excited $\tilde{B}, v^{\prime}$ levels to be isolated in the $\tilde{B}$ state and to couple individually to $\tilde{X}, v^{\prime \prime}$. Thus mode selective behaviour of $k_{n r}$, reflecting mode specific coupling matrix elements between $\tilde{B}$ and $\tilde{X}$, is reasonable to observe at small $E_{v}$.

At higher internal energies we enter region II. As $E_{v}$ increases, anharmonicity factors will become more important and $\rho\left(\tilde{B}, v^{\prime}\right)$ will also increase; more Fermi resonance and Coriolis coupling 
possibilities will result. Greater zero-order level mixing and dynamic vibrational redistribution within the $\tilde{B}$ vibrational manifold will gradually set in. The marked decrease of observed, $k_{n r}\left(1^{n} 2^{1}\right)$ with increasing $n$ as compared to the calculated values, in contrast to the behaviour of the $1^{n}$ levels, indicates that the presence of one quantum of mode 2 facilitates vibrational redistribution towards levels that have poorer Franck-Condon factors in the ENRT process.

At very high $E_{v}$ (region III) we approach a situation of a quasicontinuous set of $\tilde{B}, v^{\prime}$ levels which would correspond to the fully communicating "statistical limit" case of complete vibrational redistribution. The existence of such a continuum can be predicted for energies $E_{v}$ such that the mean energy interval $\rho^{-1}$ between levels in the $\{s\}$ vibrational manifold is smaller than the average width $\Gamma_{s}$ of these levels. The lower limit of $\Gamma_{s}$ (in $\mathrm{cm}^{-1}$ ) is $(2 \pi c \tau)^{-1}$, where $\tau$ is the lifetime observed in fluorescence and reflects only those processes which depopulate the fluorescing electronic state $S$. Vibrational redistribution certainly adds a further contribution to $\Gamma_{s}$. We can thus estimate an upper limit $E_{\text {III }}$ beyond which $\tilde{B}, v^{\prime}$ levels form a quasicontinuum. Calculating $\rho\left(\tilde{B}, v^{\prime}\right)$ by the Haarhoff approximation ${ }^{8}$ and using our measured lifetimes $\tau\left(\tilde{B}, v^{\prime}\right)$, we find that $\rho^{-1}=(2 \pi c \tau)^{-1}$ at energy $E_{\mathrm{III}}=6900 \mathrm{~cm}^{-1}$. This calculated upper limit, neglecting vibrational redistribution, is compatible with the $4500 \mathrm{~cm}^{-1}$ value estimated for the onset energy of region III, as evidenced by the quasicollinearity of the $k_{n r}\left(1^{n}\right)$ and $k_{n r}\left(1^{n} 2^{1}\right)$ values as a function of $E_{v}$ above $4500 \mathrm{~cm}^{-1}$ (Figure 7).

Figures 6 and 7 also show that there is no significant discontinuity in the $k_{n r}$ value on passage to the $\tilde{C}, 0^{\circ}$ level where the excess vibrational energy in the $\tilde{B}$ state is $10400 \mathrm{~cm}^{-1}$. This can be taken as evidence that very efficient intramolecular vibrational redistribution takes place in the vibronic levels to which the $\tilde{C}, 0^{\circ}$ state is coupled, similar to the explanation of the continuity in $k_{n r}$ found for $\beta$-naphthol and $\beta$-naphthylamine on crossing the threshold to the $S_{3}$ state. ${ }^{31}$ More details on VNRT and ENRT involving the $\tilde{C}$ state are given elsewhere.,

Relaxation processes discussed above have their analogues in models of unimolecular dissociation (UMD) reactions ${ }^{2}$ and in multiphoton dissociation. ${ }^{32}$ In both cases vibrational redistribution is essential in order to achieve the adequate nuclear configurations for transition to the dissociation continuum for example, in UMD, the RRKM 
model supposes the existence of very rapid VNRT prior to dissociation so that the whole phase space corresponding to all vibrational (and rotational) substates is equally accessible, subject only to conservation of energy and of angular momentum restraints.

Recent approaches to these problems equate the onset of rapid intramolecular vibrational redistribution with the onset of dynamical chaos, ${ }^{33}$ but no quantitative correlations have been reported for specific examples.

Parmenter ${ }^{34}$ and Smalley $^{35}$ have recently reviewed results on VNRT in excited electronic states of large neutral molecules. Three different techniques which have given information on VNRT in neutral species ${ }^{34}$ are (i) a comparison of level widths determined by high resolution absorption spectroscopy and from the fluorescence decay constant; (ii) study of the fluorescence spectrum as a function of $E_{v}$, in single vibrational level excitation experiments, where the development of a background and the broadening of spectral features are considered as indicators of the onset of VNRT processes; (iii) studies based on the time dependent development of background emission from the excited species.

For single ring aromatics without side chains, VNRT sets in at $E_{v} \sim 2000 \mathrm{~cm}^{-1} .^{34}$ For example, in the case of the $S_{1}$ state of p-difluorobenzene, Covaleskie et $a l^{36}$ have inferred that no significant VNRT occurs below $E_{v}=1600 \mathrm{~cm}^{-1}$, but that a high VNRT rate, of the order of $10^{11} \mathrm{~s}^{-1}$, exists at $E_{v}>2400 \mathrm{~cm}^{-1}$; Halberstadt and Tramer $^{37}$ also showed that at $E_{v}=2190 \mathrm{~cm}^{-1}$, the VNRT rate is greater than $3 \times 10^{9} \mathrm{~s}^{-1}$.

In our work, evidence for vibrational redistribution is obtained by a new method, (inherently also based on time-dependent phenomena since radiative and nonradiative rates act as internal clocks and probes), in which the criterion of VNRT is deviation of experimentally determined radiationless rates from values calculated with a model where vibrational redistribution is excluded. This has led us to evaluate the onset of significant VNRT in the $\tilde{B}^{2} A_{2 u}$ state of $\mathrm{C}_{6} \mathrm{~F}_{6}^{+}$to occur at $E_{v} \approx 2500 \mathrm{~cm}^{-1}$, which is of the same order of magnitude as the vibrational energy for onset of VNRT in the $S_{1}$ state of $\mathrm{C}_{6} \mathrm{H}_{6}{ }^{38}$ and $p-\mathrm{C}_{6} \mathrm{H}_{4} \mathrm{~F}_{2}{ }^{36,37}$

The model illustrated in Figure 8 suggests that VNRT in $\mathrm{C}_{6} \mathrm{~F}_{6}^{+}$ could also be followed by time and energy dependent spectral analysis of the fluorescence. Emission from unrelaxed (insignificant VNRT) $s$ 
levels, should have a different spectral distribution than the emission from relaxed levels. Such observations require a different experimental approach than the T-PEFCO method where the number of fluorescence photons produced is at present too small for dispersed spectra studies.

\section{References}

1. K. F. Freed, Top. Appl. Phys. 15, 23 (1976); P. Avouris, W. M. Gelbart and M. A. El Sayed, Chem. Revs. 77, 793 (1977); A. Tramer and R. Voltz, in: Excited States, Vol. 4 (ed. E. C. Lim. Academic Press, New York, 1979) p. 281; Radiationless Transitions, ed. S. H. Lin (Academic Press, New York, 1980).

2. H. M. Rosenstock, Adv. Mass. Spectrom. 4, 523 (1968); P. J. Robinson and K. A. Holbrook, Unimolecular Reactions (Wiley, New York, 1972).

3. S. Leach, G. Dujardin and G. Taïeb, J. Chim. Phys. 77, 705 (1980).

4. J. P. Maier and F. Thommen, Chem. Phys. 57, 319 (1981).

5. G. Dujardin and S. Leach, J. Chem. Phys. (1983) in press.

6. G. Dujardin and S. Leach, J. Chem. Soc. Faraday Disc. No. 75 (1983).

7. J. H. D. Eland, M. Devoret and S. Leach, Chem. Phys. Lett. 43, 97 (1976).

8. G. Dujardin, S. Leach and G. Taïeb, Chem. Phys. 46, 407 (1980).

9. M. Bloch and D. W. Turner, Chem. Phys. Lett. 30, 344 (1975).

10. J. P. Maier and F. Thommen, Chem. Phys. 51, 319 (1980).

11. E. W. Schlag, R. Frey, B. Gotchev, W. B. Peatman and H. Pollack, Chem. Phys. Lett. 51, 406 (1977).

12. G. Dujardin, S. Leach, O. Dutuit, T. Govers and P. M. Guyon, J. Chem. Phys. (1983) in press.

13. T. Baer in Gas Phase Ion Chemistry, Vol. 1, ed. M. T. Bowers (Academic, New York, 1979, p. 153.)

14. T. Baer, P. M. Guyon, I. Nenner, T. R. Govers, A. Tabché-Fouhaillé, R. Botter and L. F. A. Ferreira, J. Chem. Phys. 70, 1585 (1979).

15. See e.g., H. Okabe, Photochemistry of Small Molecules (Wiley Interscience, New York, 1978).

16. S. Leach in Photophysics and Photochemistry in the Vacuum Ultraviolet (NATOASI, Lake Geneva, Wisconsin, 1982), in press.

17. D. Gauyacq, M. Horani, S. Leach and J. Rostas, Can. J. Phys. 53, 2040 (1975);

D. Gauyacq, C. Larcher and J. Rostas, Can. J. Phys. 57, 1634 (1979); C. Larcher, D. Gauyacq and J. Rostas, J. Chim. Phys. 77, 655 (1980); C. Larcher, Thesis, Université de Paris-Sud, Orsay, 1982.

18. J. Rostas and R. P. Tuckett, J. Mol. Spectrosc. 96, 77 (1982); M. A. Johnson, J. Rostas and R. N. Zare, Chem. Phys. Lett. 92, 225 (1982).

19. S. Leach, M. Devoret and J. H. D. Eland, Chem. Phys. 33, 113 (1978); S. Leach, P. R. Stannard and W. M. Gelbart, Mol. Phys. 36, 1119 (1978).

20. M. A. Johnson, R. N. Zare, J. Rostas and S. Leach, manuscript in preparation.

21. G. Dujardin, S. Leach, G. Taïeb, J. P. Maier and W. M. Gelbart, J. Chem. Phys. 73, 4987 (1980).

22. A. Nitzan and J. Jortner, J. Chem. Phys. 55, 1355 (1971).

23. C. Cossart-Magos, D. Cossart and S. Leach, Mol. Phys. 37, 793 (1979).

24. G. Dujardin and S. Leach, Chem. Phys. Lett. 96, 337 (1983).

25. K. G. Spears and S. A. Rice, J. Chem. Phys. 55, 5561 (1971). 
26. I. H. Kühn, D. F. Heller and W. M. Gelbart, Chem. Phys. 22, 435 (1977).

27. A. Amirav, U. Even and J. Jortner, Chem. Phys. Lett. 71, 12 (1980).

28. P. R. Stannard and W. M. Gelbart, J. Phys. Chem. 85, 3592 (1981).

29. C. Tric. Chem. Phys. 14, 189 (1976).

30. K. F. Freed and A. Nitzan, J. Chem. Phys. 73, 4765 (1980).

31. J. C. Hsieh, C. S. Huang and E. C. Lim, J. Chem. Phys. 60, 4345 (1974).

32. R. V. Ambartzumian and V. S. Letokhov, in: Chemical and Biochemical Applications of Lasers, Vol. III, ed. C. B. Moore (Academic Press, New York, 1977) p. 167.

33. See e.g., E. J. Heller and M. J. Davis, J. Phys. Chem. 86, 2118 (1982); S. A. Rice and R. Kosloff, J. Phys. Chem. 86, 2153 (1982); W. P. Reinhardt, J. Phys. Chem. 86, 2158 (1982); P. Pechukas, J. Phys. Chem. 86, 2239 (1982).

34. C. S. Parmenter, J. Phys. Chem. 86, 1736 (1982).

35. R. E. Smalley, J. Phys. Chem. 86, 3504 (1982).

36. R. A. Covaleskie, D. A. Dolson and C. S. Parmenter, J. Chem. Phys. 72, 5774 (1980).

37. N. Halberstadt and A. Tramer, J. Chem. Phys. 73, 6343 (1980).

38. A. E. W. Knight, C. S. Parmenter and M. W. Schuyler, J. Am. Chem. Soc. 97, 1993 (1975). 